\title{
Improved survival in critically ill patients: are large RCTs more useful than personalized medicine? We are not sure
}

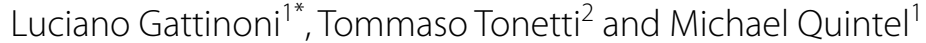

C 2016 Springer-Verlag Berlin Heidelberg and ESICM

At first sight, personalized medicine and large randomized clinical trials (RCTs) seem to reflect opposite approaches to medicine: one focusing on the single individual, the other on the population of individuals. Accordingly, in critically ill patients, does the "best" therapy depend on the unique characteristics of the patient or does it derive from the outcome analysis of a large population in which a given therapy has been tested? Actually, we believe that comparing personalized medicine and large RCTs is like comparing apples and oranges. Indeed, which are the essences of personalized medicine and which those of the RCTs?

As defined in the Oxford Dictionary of English [1], personalized medicine is "a type of medical care in which treatment is customized for an individual patient". In this sense, we are simply referring to the basic normal medical practice since Hippocrates: we cure a patient, not a population. Within this general statement, a first step to better treat an individual patient (personalization) was (and is) to tailor the treatment according to challenge tests, such as steroid administration after a corticotropin test in septic patients [2] or volume replacement after a fluid challenge [3] or the positive end-expiratory pressure (PEEP) setting after a recruitability assessment [4]. A further step towards the personalized medicine could be the definition of patient clusters or subgroups which better reflect pathophysiological differences and treatment options [5]. The ideal final step should be to customize

\footnotetext{
*Correspondence: gattinoniluciano@gmail.com

${ }^{1}$ Department of Anesthesiology, Emergency and Intensive

Care Medicine, University of Göttingen, Robert-Koch-Straße 40,

37075 Göttingen, Germany

Full author information is available at the end of the article
}

For contrasting viewpoints, please go to doi: 10.1007/s00134-016-4482-5 and doi: 10.1007/s00134-016-4491-4. the medical treatment according to the individual molecular/genetic setup ("precision medicine"). Therefore, in general, "personalized" medicine attempts to better define the patients' characteristics through progressively smaller subgroups, down to the level of the individual genome. Ideally, at the asymptote of this process, each patient will receive a therapy unique to him, with maximal efficacy and minimal side effects. In other words, with the ultimate personalized medicine, the problems due to population heterogeneity will not exist anymore.

The large RCTs are instead not a particular approach to medicine but are just experiments. Mostly, they are designed to find whether a given treatment provides benefits compared to another one. To fully understand the essence of these experiments, however, a few considerations are needed. First, a solid background, derived from experience, observations and physiology, is necessary. From this background, a hypothesis is generated. The $\mathrm{RCT}$ is the experiment designed to prove (or disprove) the hypothesis. If the hypothesis is confirmed, the theory is valid; if it is rejected, the theory is wrong. Therefore, the essence of the RCTs is to provide "unquestionable" evidence that a given theory is valid. This occurs if the theory generates a treatment hypothesis which is proved beneficial.

If these are the essences of the personalized medicine and of the RCTs, it is quite clear that, in theory, they are complementary and equally necessary, as summarized in Fig. 1. As shown, the progressive increase in "personalization" allows the defining of more specific groups and subgroups of patients for whom a given treatment, finally, is proved to be beneficial. The asymptote of this process will be-in an ideal world-a degree of personalization so high as to concern a single individual with a therapy unique to him. 


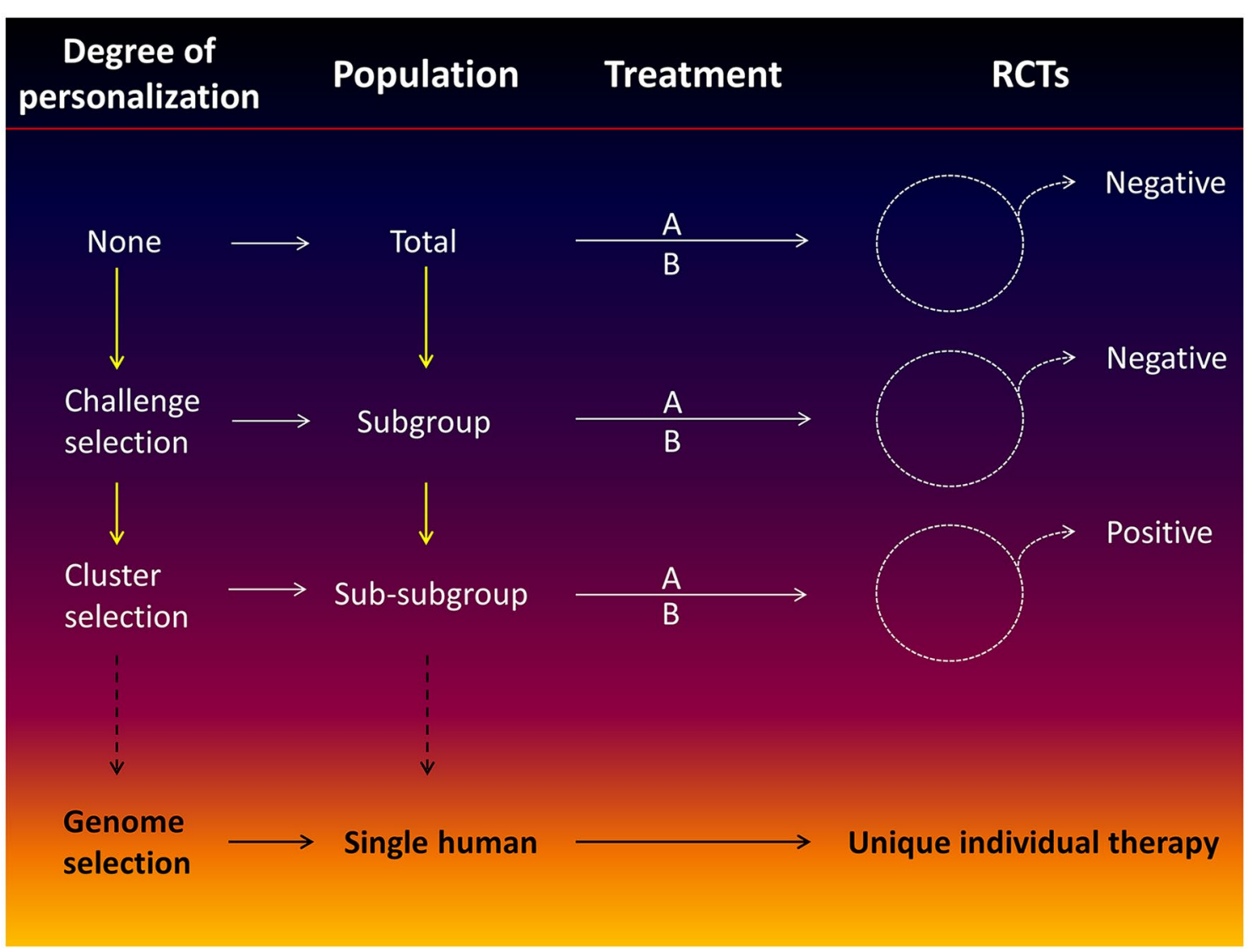

Fig. 1 Increasing the degree of personalization (none, challenge test, cluster definition, genome analysis) allows the progressive decreasing of the heterogeneity of the sample. Accordingly, the chance of success in testing a given treatment in a selected population increases. At present, most of the trials are conducted at a degree of personalization equal to "none". The asymptote of the process of personalized medicine (precision medicine) will ideally be a unique therapy for a single patient, through genome analysis

All this in theory; but what happens in our real life? Most of the treatments tested through RCTs have been performed in a general population of critically ill patients, presenting not with the disease but with a syndrome. The two main paradigms are sepsis and the acute respiratory distress syndrome (ARDS). The theory behind most of the RCTs applied to septic populations is that the sepsis syndrome is caused by a disproportionate/dysregulated inflammatory response of the host. This theory generated the hypothesis that the control of the inflammatory reaction may be of benefit. Accordingly, hundreds of anti-inflammatory treatments (corticosteroids, cytokine antagonists, receptor inhibitors, statins, etc.) have been tested in the last 30 years. All the trials were negative, disproving the hypothesis. The obvious conclusion is that the theory of disproportionate inflammatory response, if applied to the total septic population, is wrong.

Observations, experience and physiological background have told us that an imbalance between oxygen supply and consumption could lead, with time, to multi-organ dysfunction/failure. The hypothesis generated from this theory is that a proper and early correction of the imbalance, indicated by a normalization of the mixed venous saturation, should be of benefit. Actually, the hypothesis was proved right in patients in whom the imbalance was present [6]. However, when applied in RCTs on total septic populations, where the imbalance was not present in most of the patients, no benefit could be demonstrated [7-9]. Unfortunately, in most of the studies carried out in critically ill patients, the degree of personalization was "none" [10]. Further steps are necessary.

The bases of some degree of personalization in critically ill patients are undoubtedly already present. This is particularly true, referring to Fig. 1, for a degree of personalization defined by the response to a challenge test. As an example, there have been several suggestions that the value of PEEP during ARDS should be tailored according to the severity of the syndrome. Most of the "challenge" studies were physiological and identified subgroups of 
patients for which a given treatment could be of benefit. Unfortunately, even this first step of the personalization did not translate into RCTs.

Genome-wide association studies (GWAS), in general, try to find the association between outcome (or other phenomena, such as response to drugs) and specific variants at the genomic level. If associations are found, it is possible either to better personalize the treatment or to identify potential novel targets for therapy, prevention or development of biomarkers for risk stratification. While the GWAS approach is increasingly used in oncology [11, 12], in critical care it is still in its infancy and primarily confined to sepsis research. In sepsis due to pneumonia, Rautanen and colleagues identified a strong association between common variants in the FER gene and reduced risk of death [13]. Christaki and Giamarellos-Bourboulis recently reviewed the issue, concluding that a more personalized approach based on genomics will be possible in the next decade [14]. Similar conclusions were reached by Pinheiro da Silva and Cerquiera César Machado, discussing the actual status of personalized medicine for sepsis [15].

In conclusion, both personalized medicine and RCTs are, in theory, beautiful instruments for increasing our knowledge. As for which instrument, however, the results will depend on how they are used and integrated. At present, there is a very large space for improvement in the personalization of treatment, even without genomics, as the basis for a more meaningful use of RCTs.

\footnotetext{
Author details

${ }^{1}$ Department of Anesthesiology, Emergency and Intensive Care Medicine, University of Göttingen, Robert-Koch-Straße 40, 37075 Göttingen, Germany. ${ }^{2}$ Department of Medicine, Institute of Anesthesiology and Intensive Care Medicine, University of Padova, Padova, Italy.
}

\section{Compliance with ethical standards}

\section{Conflicts of interest}

On behalf of all authors, the corresponding author states that there is no conflict of interest.
Received: 22 July 2016 Accepted: 28 July 2016

Published online: 12 September 2016

\section{References}

1. Stevenson A (ed) (2010) Oxford Dictionary of English (3rd edn). Oxford University Press, Oxford

2. Annane D et al (2002) Effect of treatment with low doses of hydrocortisone and fludrocortisone on mortality in patients with septic shock. JAMA 288(7):862-871

3. Pinsky MR (2015) Functional hemodynamic monitoring. Crit Care Clin 31(1):89-111

4. Chiumello D et al (2015) Lung recruitment assessed by respiratory mechanics and by CT scan: what is the relationship? Am J Respir Crit Care Med 193:1254-1263

5. Knox DB et al (2015) Phenotypic clusters within sepsis-associated multiple organ dysfunction syndrome. Intensive Care Med 41(5):814-822

6. Rivers E et al (2001) Early goal-directed therapy in the treatment of severe sepsis and septic shock. N Engl J Med 345(19):1368-1377

7. Pro Cl et al (2014) A randomized trial of protocol-based care for early septic shock. N Engl J Med 370(18):1683-1693

8. Peake SL et al (2015) Goal-directed resuscitation in septic shock. N Engl J Med 372(2):190-191

9. Mouncey PR et al (2015) Trial of early, goal-directed resuscitation for septic shock. N Engl J Med 372(14):1301-1311

10. Gattinoni L, Giomarelli P (2015) Acquiring knowledge in intensive care: merits and pitfalls of randomized controlled trials. Intensive Care Med $41(8): 1460-1464$

11. Van Allen EM, Wagle N, Levy MA (2013) Clinical analysis and interpretation of cancer genome data. J Clin Oncol 31(15):1825-1833

12. Low SK et al (2014) Genome-wide association study: a useful tool to identify common genetic variants associated with drug toxicity and efficacy in cancer pharmacogenomics. Clin Cancer Res 20(10):2541-2552

13. Rautanen A et al (2015) Genome-wide association study of survival from sepsis due to pneumonia: an observational cohort study. Lancet Respir Med 3(1):53-60

14. Christaki E, Giamarellos-Bourboulis EJ (2014) The beginning of personalized medicine in sepsis: small steps to a bright future. Clin Genet 86(1):56-61

15. Pinheiro da Silva F, Cesar Machado MC (2015) Personalized medicine for sepsis. Am J Med Sci 350(5):409-413 